\title{
MEASUREMENT OF SMALL BEAM SIZE BY THE USE OF SR INTERFEROMETER
}

\author{
S. HIRAMATSU, H. IWASAKI*, T. MITSUHASHI, T. NAITOH and Y. YAMAMOTO* \\ High Energy Accelerator Research Organisation, Oho, Tsukuba, Ibaraki, 305-0801 Japan \\ *SR center, Ritsumeikan University, 1916 Noji-cyo, Kusatsu, Shiga, 525-77 Japan
}

\begin{abstract}
The principle of measurement of the profile or size of small objects through the spatial coherency of the light is known as the van Citterut-Zernike theorem. Recently the SR interferometer (interferometer for synchrotron radiation) was developed to measure the spatial coherency of the visible region of the SR beam, and we demonstrated that this method is able to measure the beam profile and size. Since the small electron beam emits a SR beam which has a good spatial coherency, this method is suitable for measuring a small beam size. In this paper, the basic theory for the measurement of the profile or size of a small object by the spatial coherency of the light, a design of the SR interferometer, and the results of beam profile measurement are described. Two examples of the small beam size measurements are also described.
\end{abstract}

\section{INTRODUCTION}

The measurements of beam profile and size are two of the most fundamental diagnostics in an electron storage ring. The most conventional method to observe the beam profile is known as a beam profile monitor via imaging of the visible SR beam[1]. The resolution of this monitor is generally limited by diffraction phenomena. In the usual configuration of the profile monitor the RMS size of diffraction ( $1 \sigma$ of the point spread function) is no smaller than $50 \mu \mathrm{m}$. In the last 10 years, research and development in electron storage rings (especially in the area of emittance reduction) has been very remarkable. We can realise sub-diffraction-limited beam sizes in electron storage rings. So the above-mentioned profile monitor via imaging of the visible SR beam becomes useless in precise quantitative measurements of the beam profile and size. In the visible optics, opticians use an interferometer as the standard method to measure the profile or size of very small objects. The principle of measurement of the profile of an object by means of spatial coherency was first proposed by H.Fizeau [2] and is now known as the Van Cittert-Zernike theorem [3]. It is well known that A. A. Michelson measured the angular dimension (extent) of a star with this method [4]. Recently we developed the SR interferometer (an interferometer for SR beams) to measure the spatial coherency of the visible region of an SR beam, and as one of the results of investigations on the spatial coherence of

e-mail: mitsuhas@mail.kek.jp
SR beams, we demonstrated that this method is applicable to measure the beam profile and size at the KEK Photon Factory [5]. Since the SR beam from a small electron beam has good spatial coherency, this method is suitable for measuring a small beam size. The characteristics of this method are: 1) we can measure beam sizes as small as $5 \mu \mathrm{m}$ with $1 \mu \mathrm{m}$ resolution in a nondestructive manner; 2) the profile is easy to measure using visible light (typically $500 \mathrm{~nm}$ ); 3) the measurement time is a few seconds for size measurement and few tens of seconds for profile measurement. In this paper we describe the van Cittert-Zernike theorem, the design of the SR interferometer and examples of the profile and the beam size measurements

\section{SPATIAL COHERENCE AND BEAM SIZE}

According to van Citterut-Zernike's theorem, the profile of an object is given by the Fourier Transform of the complex degree of spatial coherence at longer wavelengths as in the visible light[3][6][8]. Let $f$ denotes the beam profile as a function of position $y, R$ denotes distance between source beam and the double slit, and $\gamma$ denotes the complex degree of spatial coherence as a function of spatial frequency $v$. Then $\gamma$ is given by the Fourier transform of $f$ as follows;

$$
\gamma(v)=\int f(y) \exp (-2 \pi i v \cdot y) d y, \quad v=\frac{2 \pi \mathrm{D}}{\lambda R} .
$$

We can measure the beam profile and the beam size via spatial coherence measurement with the interferometer.

\section{SR INTERFEROMETER}

To measure the spatial coherence of SR beams, a wavefront-division type of two-beam interferometer using polarized quasi-monochromatic rays was designed as shown in Fig.1.

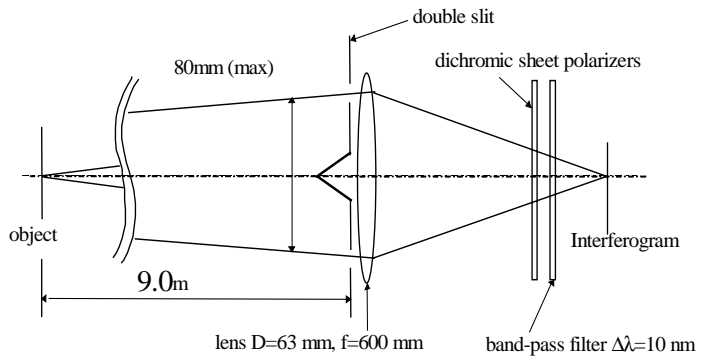

Fig.1 Outline of the SR interferometer. 
In the vertical plane, the elliptical polarity of synchrotron radiation is opposite that in between the medium plane of the electron beam orbit. Therefore, there exists the $\pi$ phase difference between the phases of the interferograms to correspond to the $\sigma$ - and $\pi$-polarized components [12]. To eliminate the interferogram by $\pi$ polarized component, we mast apply a polarization filter. A typical interferogram observed with the SR interferometer is shown in Fig.2.

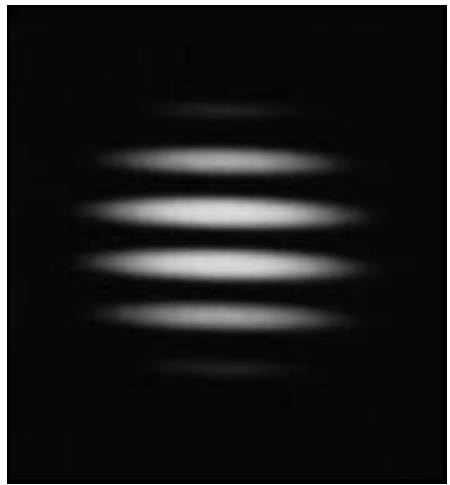

Fig. 2 A typical interferogram observed with the SR interferometer.

\section{Convenient formulas for a Gaussian approximation of the beam profile}

We often approximate the beam profile with a Gaussian shape. With this approximation, we can skip any phase measurement. The Fourier transform of even function (Gaussian) is simplified to a Fourier cosine transform. A spatial coherence is also given by a Gauss function. We can evaluate a RMS width of spatial coherence by using q least-squares analysis. The RMS beam size $\sigma_{\text {beam }}$ is given by the RMS width of the spatial coherence curve $\sigma_{\gamma}$ as follows:

$$
\sigma_{\text {beam }}=\frac{\lambda \cdot R}{2 \cdot \pi \cdot \sigma_{\gamma}}
$$

where $R$ denotes the distance between the beam and the double slit. We can also estimate the RMS. beam size

$$
\sigma_{\text {beam }}=\frac{\lambda \cdot F}{\pi \cdot D} \cdot \sqrt{\frac{1}{2} \cdot \ln \left(\frac{1}{\gamma}\right)}
$$

from one data of visibility, which is measured at a fixed separation of double slit. The RMS beam size $\sigma_{\text {beam }}$ is given by ,

where $\gamma$ denotes the visibility, which is measured at a double slit separation of $\mathrm{D}$.

\section{5 . BEAM PROFILE MEASUREMENT}

We can measure the beam profile by Fourier transform of the spatial coherence. Figure 3 shows the absolute value the complex degree of the spatial coherence ( $|\gamma|$,visibility ) was measured by changing the double slit separation from $5 \mathrm{~mm}$ to $15 \mathrm{~mm}$ at the Photon Factory[5].

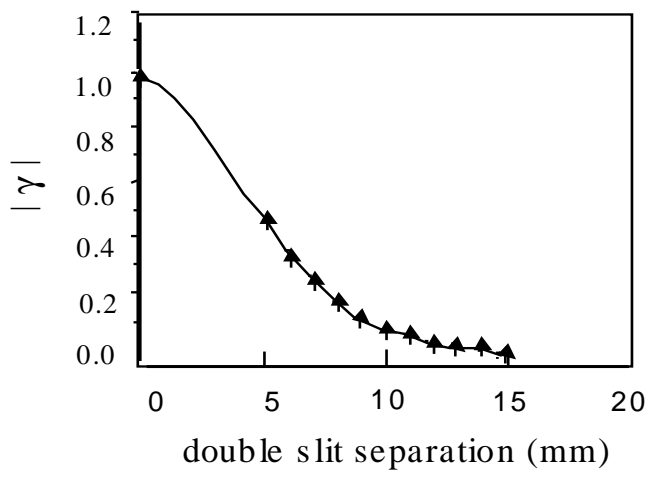

Fig 3. Result of $|\gamma|$ at the Photon Factory

The result of beam profile by Fourier transform of the spatial coherence is shown Fig. 4.

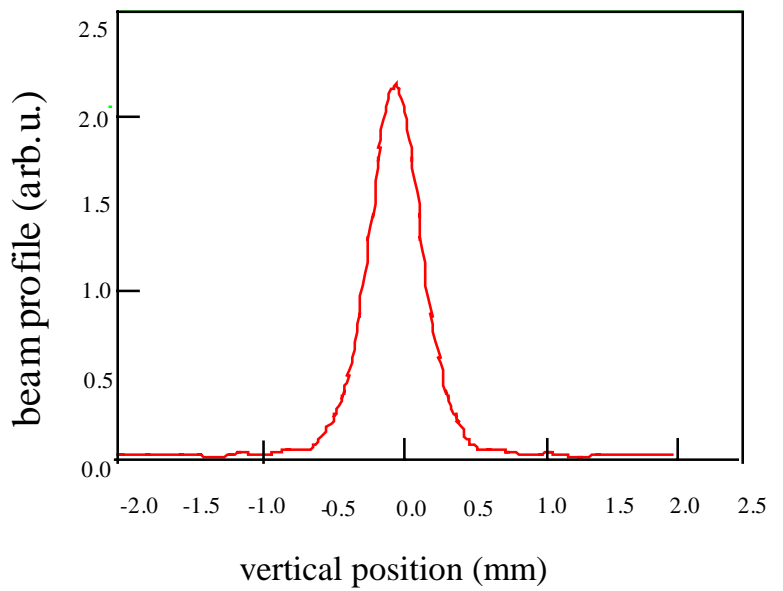

Fig. 4 Beam profile by the Fourier transform of the spatial coherence at the Photon Factory

Another example of beam profile measurement was performed at the SR facility AURORA of Ritsumeikan university[6]. Figure 5 shows the result of the $|\gamma|$ which is measured for the large beam operation mode. In this operation mode, a fat beam is obtained by a RF kicker.

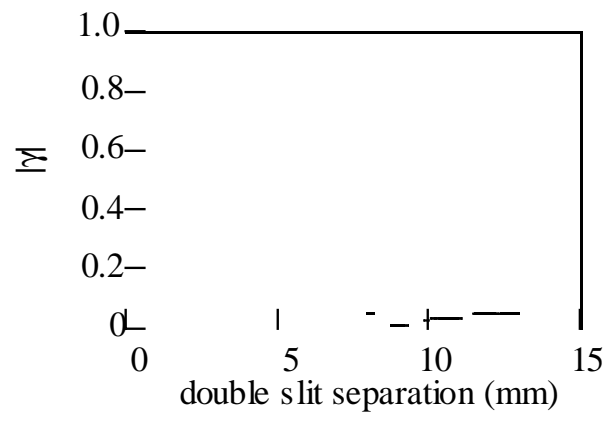

Fig. 6 Result of the $|\gamma|$ which is measured for the large beam operation mode

The result of beam profile by the Fourier transform of the $|\gamma|$ is Fig. 6. The trapezoidal profile of beam was observed due to the fat beam which is obtained by the use of RF kicker. 


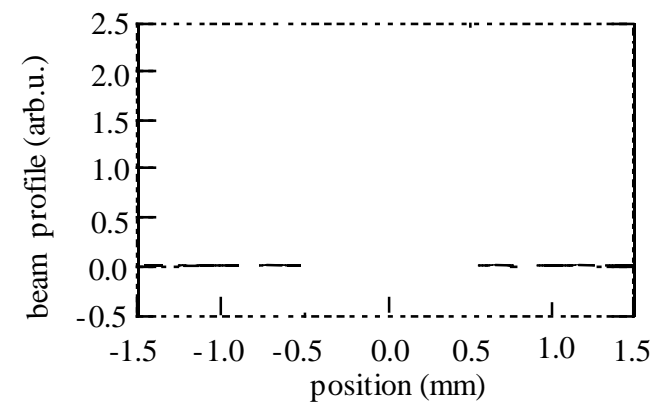

Fig. 6 The result of beam profile by the Fourier transform at the AURORA.

\section{SMALL BEAM SIZE MEASUREMENT}

As mentioned in section 3, to assume the Gaussian profile, we can evaluate the beam size in the small-beam based on the degree of spatial coherence. We introduce two examples of small beam size measurements in this section. One is the result of vertical beam size measurement at the AURORA[6] and other is the vertical and the horizontal beam size measurement the ATF damping ring at KEK[7]. Figure 7 shows the result of a least-squares fitting of the $|\gamma|$ by a Gaussian profile at the AURORA. The obtained beam size from this fitting is $16.5 \mu \mathrm{m}$.

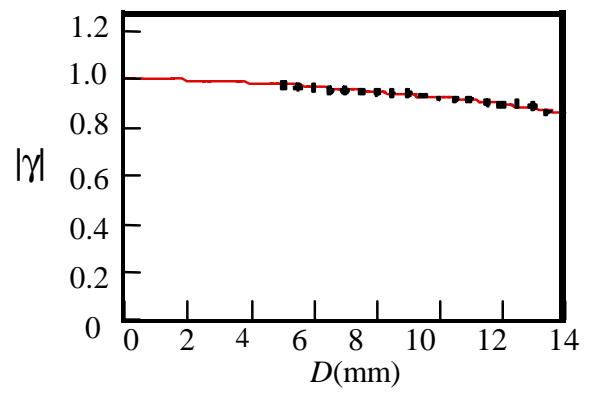

Fig. 7 Absolute value of the complex degree of spatial coherence in the vertical direction. Dotted line denotes measured $|\gamma|$, and solid line denotes the best-fit beam size of $16.5 \pm 0.6 \mu \mathrm{m}$.

Figures 8 show the result of a least-squares fitting of the $|\gamma|$ by a Gaussian profile at the ATF damping ring for vertical direction.

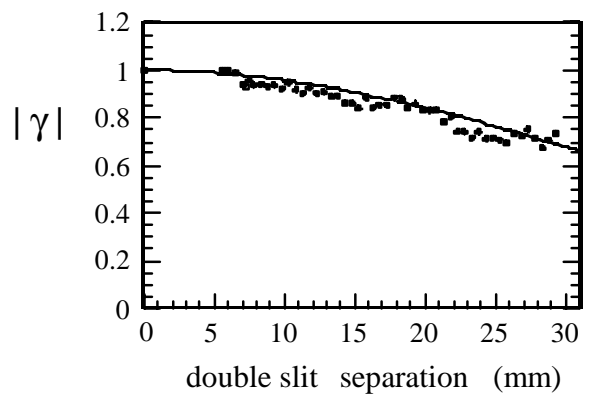

Fig. 8. Absolute value of the complex degree of spatial coherence in the vertical direction at ATF damping ring. Dotted line denotes measured $|\gamma|$, and solid line denotes the best-fit beam size of $14.7 \pm 0.6 \mu \mathrm{m}$.
A least-squares fitting of the $|\gamma|$ having a field depth effect for the horizontal direction is shown in Fig. 9. The obtained beam size from these fitting is $14.7 \mu \mathrm{m}$ in the vertical and $39 \mu \mathrm{m}$ in the horizontal.

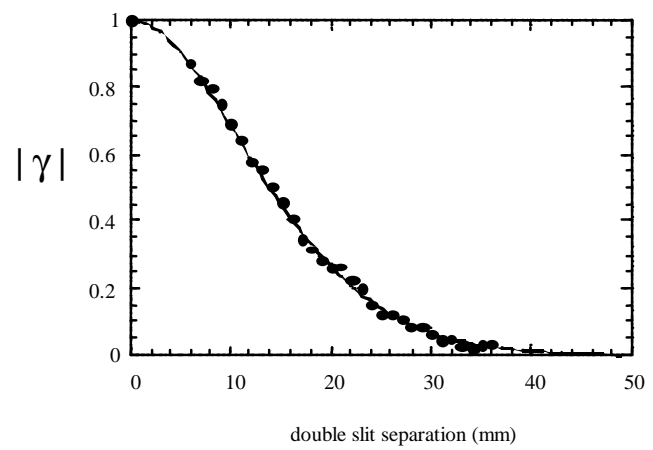

Fig. 9. Absolute value of the complex degree of spatial coherence in the horizontal plane at the ATF damping ring. The dotted line denotes measured $|\gamma|$ and the solid line denotes the best-fit value of $39 \pm 1 \mu \mathrm{m}$.

\section{CONCLUSIONS}

The SR interferometer was developed to measure the spatial coherency of the visible region of the SR beam, and we demonstrated that this method is able to measure the beam profile based on the van Cittert-Zernike theorem. Since the small electron beam emits a SR beam which has a good spatial coherency, this method is suitable for measuring a small beam size. We succeed to measure the small beam sizes of a $16.5 \mu \mathrm{m}$ at the AURORA, and $14.7 \mu \mathrm{m}$ at ATF damping ring with resolution less than $1 \mu \mathrm{m}$. The measuring time is typically about $1 \mathrm{sec}$ for beam size measurement, is about $1 \mathrm{~min}$ for profile measurement with slit scanning.

\section{ACKNOWLEDGMENTS}

The authors wish to thank to Professors M. Kihara, Y. Yamazaki and S. Kurokawa of KEK for their encouragement of this work. The authors also thank to Prof. Kobayakawa of Nagoya university for his helpful discussion.

\section{REFERENCES}

[1] T. Mitsuhashi and M. Katoh, Proc. 5th European Particle Accelerator Conference, 1669, (1996), Sitges (Barcelona).

[2] H. Fizeau, C.R.Acd.Sci. Paris, 66, 934 (1868).

[3] P.H.van Cittert, Phisica, 1, 201(1934) and also see M. Born and E. Wolf, "Principles of Optics", chapter 10, P491, Pergamon press. (1980).

[4] A.A. Michelson, Astrophsyc.J51,257 (1920).

[5] T. Mitsuhashi, Proc of 1997 Particle Accelerator Conference, 766, (1997), Vancouver.

[6] T. Mitsuhashi, H. Iwasaki,Y. Yamamoto,

T. Nakayama and D. Amano, Proc. 11th Symposium on Accelerator Science and Technology 441 (1997).

[7] T. Mitsuhashi and T. Naitoh, Proc.of 6th European Particle Accelerator Conference 1565,(1998), Stockholm. 